\title{
Für einen empirischen Ideologiebegriff. Ein wissenssoziologischer Blick auf die Ideologiekritik
}

Birgit Peuker

Keywords: Wissenschaftstheorie, Ideologiekritik, Soziologie wissenschaftlichen Wissens, Wissenssoziologie

Abstract

In dem Beitrag werden Bezüge zwischen Ideologiekritik und der Wissenschaftstheorie der Sozialwissenschaften hergestellt. Die methodologischen Herausforderungen, die sich insbesondere der Soziologie wissenschaftlichen Wissens stellen, können ebenso für eine Ideologiekritik als sozialwissenschaftliche Praxis gelten. Aporien, die sich verschiedenen Ideologietheorien im Laufe der Entwicklung des Ideologiebegriffs stellen, können so aufgeklärt werden. Darüber hinaus wird in dem Beitrag dafür plädiert, den Begriff der Ideologie auf eine spezifische Wissensform zu beschränken und nicht auf die gesellschaftlichen Bedingungen auszudehnen, die zu ihrer Entstehung führen. Ideologiekritik ist damit nicht in eine Ideologietheorie, sondern in eine Gesellschaftstheorie einzubetten. Ideologien können dann als eine Wissensform in der Nähe von Macht und Herrschaft empirisch untersucht werden, um Wissensformen als mehr oder weniger ideologisch kennzeichnen zu können.

Peuker, Birgit. 2019. „Für einen empirischen Ideologiebegriff. Ein wissenssoziologischer Blick auf die Ideologiekritik“. In Ideologie, Kritik, Öffentlichkeit. Verhandlungen des Netzwerks Kritische Kommunikationswissenschaft, herausgegeben von Uwe Krüger und Sebastian Sevignani, 86-101. Universität Leipzig. DOI: 10.36730/ideologiekritik.2019.5

Birgit Peuker | Freie Universität Berlin | birgit.peuker@fu-berlin.de 


\section{Einführung}

In der Gesellschaft gibt es viele Meinungen, Positionen und Wissensansprüche, die sich widersprechen. Ein Beispiel aus der Umwelt- und Techniksoziologie sind die Technikkonflikte um Atomkraft und Gentechnik. Hier stehen sich bei den verschiedenen Diskurspositionen Bewertungen der Techniken teilweise in Pro-Kontra-Argumentationen gegenüber. ${ }^{1}$ Dabei spielt weniger eine Rolle, welche der beiden Seiten recht hat, sondern auch, welche Seite ihre Position gegenüber der anderen durchsetzen kann.

Die Frage ist, inwiefern der Ideologiebegriff dazu geeignet sein kann, über die Macht der jeweiligen Diskursposition aufzuklären. Die Antwort, die der Artikel gibt, ist: Ja, wenn Ideologien als ein Machtmittel unter vielen analysiert werden. Der Ideologiebegriff sollte hierzu auf eine bestimmte Wissensform in der Nähe von Macht und Herrschaft fokussiert und Ideologien im Zusammenhang mit nichtsprachlichen Machtmitteln untersucht werden. Ideologiekritik sollte von der Ideologietheorie getrennt werden. ${ }^{2}$ Dabei wird dafür plädiert, dass sich die Ideologiekritik als eine empirische Sozialwissenschaft versteht.

Zunächst wird jedoch in dem Artikel der These nachgegangen, dass der Ideologiebegriff in der materialistischen und poststrukturalistischen Theoriebildung derart ausgeweitet wurde, dass wissenschaftstheoretische Probleme und die gesellschaftlichen Bedingungen, die für die Entstehung von Ideologien verantwortlich sein sollen, unter den Begriff gefasst wurden. Damit wurde der Begriff überdehnt und dies in zwei Di-

1 Die Überlegungen in diesem Artikel und die Beschäftigung mit dem Ideologiebegriff habe ich im Nachgang zu meiner Dissertation angestellt (vgl. Peuker 2010). Die dort durchgeführte Diskursanalyse des Technikkonflikts um die Agrar-Gentechnik warf insbesondere in Hinblick auf die Macht und Durchsetzungskraft unterschiedliche Diskurspositionen Fragen auf.

2 Rehmann (2008) tritt dafür ein, Ideologiekritik und Ideologietheorie zusammen zu denken. Ohne Kritik würden Ideologietheorien Gefahr laufen, „sich funktionalistisch in einverständige Legitimationstheorie zurückzuverwandeln" (Rehmann 2008, 14). Demgegenüber spreche ich mich für eine Herauslösung der Ideologiekritik oder der Kritik an Ideologien aus der Ideologietheorie aus. Der - kritisch gebrauchte - Begriff sollte besser in eine umfassendere Gesellschaftstheorie oder in Theorien mittlerer Reichweite, die auf diese hinausweisen, eingebettet werden, um so die vielfältigen Bezüge, die ideologische Wissensformen hervorbringen, empirisch zu untersuchen. 
mensionen: einmal hinsichtlich der Epistemologie und einmal hinsichtlich der gesellschaftlichen Bedingungen von Ideologien. Basis für die folgenden Ausführungen bilden Sekundäranalysen zur Entwicklung der Ideologietheorie unter wahlweisem Bezug auf Primärliteratur. ${ }^{3}$

Im ersten Abschnitt wird dargelegt, dass es grundlegend notwendig ist, zwischen Ideologiekritik als einem sozialwissenschaftlichen Ansatz und ihrem Gegenstand, Ideologien, zu unterscheiden. Hierbei wird deutlich, dass Ideologiekritik als Untersuchung und Bewertung von Ideologien Probleme mit sich bringt, die ebenso in der Wissenschaftstheorie der Sozialwissenschaft diskutiert werden. Diese Probleme sollten nicht als dem Ideologiebegriff inhärent aufgefasst werden.

Im zweiten Abschnitt wird diskutiert, in welchem Verhältnis Ideologien als spezifische Wissensform zu den gesellschaftlichen Bedingungen stehen, die sie hervorbringen. Dabei wird dafür plädiert, die gesellschaftlichen Bedingungen für die Entstehung von Ideologien nicht unter den Begriff Ideologie zu fassen.

\section{Zirkularität sozialwissenschaftlicher Erkenntnis und Ideologiekritik}

Ideologiekritik kann als eine sozialwissenschaftliche Methode aufgefasst werden, die Ideologien als eine bestimmte Wissensform in der Gesellschaft zum Gegenstand hat. Damit stellen sich der Ideologiekritik methodologische Probleme, die generell die sozialwissenschaftliche Forschung kennzeichnen. Diese Probleme wurden in der Reflexion der Ideologietheorie jedoch teilweise als Aporien des Ideologiebegriffs selbst betrachtet.

Der sozialwissenschaftliche Forschungsprozess ist oft als zirkulär beschrieben worden. Der Forschende ist Teil dessen, was er erforscht: der Gesellschaft (vgl. Dilthey (1974 [1900]), Felt et al. 1995: 165ff.; Böhme und Potyka 1995: 19ff.). Sowohl der Forschungsprozess als auch die Forschungsergebnisse sind gesellschaftlich bedingt und haben Wirkungen

3 Für diese Durchsicht wurden folgende Werke aufgearbeitet: Eagleton 2000; Knoblauch 2010; Rehmann 2008; Ritsert 2002, Herkommer 2004. Dabei wird sich im Folgenden aus Gründen der Übersichtlichkeit vor allem auf Eagleton 2000 bezogen. 
auf gesellschaftliche und soziale Prozesse. Insbesondere bei der Untersuchung gesellschaftlicher Wissensformen wird die Zirkularität deutlich, da hier Wissen über Wissen produziert wird. Dabei kann die Forderung aufgestellt werden, dass wissenschaftliche Aussagen über Ursachen und Zusammenhänge, die für die untersuchten Wissensformen gelten sollen, ebenso für den eigenen Ansatz Gültigkeit haben, um Einheitlichkeit und Konsistenz der Theorie zu gewährleisten.

Diese Forderung kann mit David Bloor (1991), einem der Begründer des wissenssoziologischen Ansatzes der Wissenschaftssoziologie, untermauert werden. Bloor stellte an eine Wissenschaft über wissenschaftliches Wissen in seinem "strong programme“ vier Forderungen auf: Erstens sollten kausale Erklärungen für bestimmte Glaubens- und Wissensformen aufgestellt werden (Kausalität). Zweitens sollte sich den Aussagen unabhängig davon, ob sie als wahr oder falsch gelten, unvoreingenommen genähert werden (Unvoreingenommenheit). Das heißt, sowohl wahre als auch falsche Aussagen sollen erklärt werden. Drittens sollten sowohl für wahre als auch für falsche Aussagen dieselben Gründe angeführt werden (Symmetrieprinzip). Viertens sollten die Erklärungsmuster auch auf die Soziologie selbst anwendbar sein (Reflexivität).

Die Ideologiekritik weist viele Parallelen zu dem Versuch auf, wissenschaftliches Wissen zu erklären. So will sie, ganz dem Prinzip der Kausalität bei David Bloor folgend, kausale Erklärungen für bestimmte Aussagen und Wissensformen angeben. Ideologiekritik hat jedoch anders als die wissenssoziologische Wissenschaftssoziologie nicht (nur) wissenschaftliches Wissen als ihren Gegenstand, sondern gesellschaftlich produziertes Wissen im Allgemeinen.

In seiner Abhandlung über die Entwicklung der Ideologietheorie ist Eagleton (2000) mehrfach auf Probleme im Umgang mit der Zirkularität gestoßen. Drei seiner Beispiele sollen hier näher betrachtet werden.

So erläutert Eagleton (2000) in Bezug auf die Ideologiekritik der frühen Aufklärung, dass deren Vertreter das Ziel gehabt hätten, die Sinneserfahrungen des Menschen aus den materiellen Verhältnissen abzuleiten. Damit hätten sie den Verstand in die wissenschaftliche Untersuchung mit einbezogen, woraus sich die folgende Frage ergeben habe: „Wenn es jedoch das Ziel von Ideologie ist, die Ursprünge menschlichen Bewusstseins zu untersuchen, was soll man dann über das Bewusstsein dessen, der diese Untersuchungen durchführt, sagen?“ (Eagleton 2000, 84) Diese Frage verweist auf das Prinzip der Reflexivität bei Bloor (1991). Auch die 
sozialen Ursprünge der Ideologiekritik sollten durch diese selbst erklärt werden können.

Ein weiteres Beispiel lässt sich bei Eagleton (2000) in Bezug auf Karl Marx“ und Friedrich Engels" „Deutsche Ideologie“ (Marx/Engels 1953 [1857]) finden. In diesem Aufsatz arbeiten die Autoren die gesellschaftlichen Bedingungen eines "falschen Bewusstseins“ heraus. Dem liege die falsche Vorstellung zu Grunde, dass Ideen getrennt von der gesellschaftlichen Tätigkeit seien (Marx/Engels 1953 [1857], 14f. u. 46f.). Dem wird in der „Deutschen Ideologie“ die Grundthese gegenübergestellt, dass Ideen in der Praxis miteinander verflochten seien, aber sich das Bewusstsein durch die Trennung von Hand- und Kopfarbeit von den materiellen Gegebenheiten entfernen könne und damit die Ideensphäre eine relative Autonomie erreiche (Marx/Engels 1953 [1857], 28). Die Dichotomie zwischen Ideen und gesellschaftlicher Wirklichkeit ist damit ein Produkt der gesellschaftlichen Verhältnisse und die relative Autonomie von Ideen real. Ideen bleiben aber dennoch, trotz ihrer Falschheit, gesellschaftlich wirksam (Eagleton 2000: 90). Dies stellt nach Eagleton (2000) ein Paradox dar, da gesellschaftliche Illusionen in ihrem „Falschsein irgendwie strukturell notwendig für eine bestimmte Gesellschaftsordnung [sind]. Man könnte sagen, daß die Falschheit der Ideen ein Teil der ,Wahrheit' der gesamten materiellen Verhältnisse ist" (Eagleton 2000, 87). Dieses Paradoxon kann durch die Symmetrieforderung bei Bloor (1991) aufgelöst werden. Sowohl wahre als auch falsche Aussagen existieren in der gesellschaftlichen Wirklichkeit und können empirisch untersucht und einer gemeinsamen Erklärung zugeführt werden. Dabei ist die Position, aus der heraus die Analyse von Ideologien erfolgt, selbst ein Produkt der Trennung von Hand- und Kopfarbeit, insofern sie als sozialwissenschaftliche Praxis verortet ist.

Weiterhin wird in der "Deutschen Ideologie“ ebenso deutlich, dass unter dem Ideologiebegriff auch die Bedingungen gefasst werden sollen, die ideologische Wissensformen erst hervorbringen. So stellt sich in diesem Zusammenhang die Frage: Ist die Trennung von Hand- und Kopfarbeit schon Ideologie oder trägt sie nur zur Entstehung von Ideologien bei? Ist jegliches durch Wissensarbeiter produziertes Wissen Ideologie? Die Bedingungen für die Entstehung von Ideologie sollten durch die Theorie geklärt werden, in die der Ideologiebegriff eingebettet ist. Werden die Bedingungen für ihre Entstehung unter den Ideologiebegriff subsumiert, kann nur ungenügend zwischen mehr oder weniger ideologischem Wissen empirisch unterschieden werden. 
Direkt auf die Zirkularität bei der wissenschaftlichen Untersuchung gesellschaftlicher Wissensformen nimmt Eagleton (2000) bei seiner Diskussion von Georg Lukács Bezug. Hier besteht das Problem, wie die besondere Erkenntnisposition des Proletariats im Vergleich zum Bürgertum begründet werden könnte, da doch eigentlich jede Wissensposition durch ihren sozialen Standpunkt verzerrt sein müsste. Eagleton (2000) interpretiert Lukács Position in "Geschichte und Klassenbewusstsein" so, dass das Proletariat im Unterschied zum bürgerlichen Bewusstsein in der Lage sei, die gesellschaftliche Totalität aus dem Eigeninteresse heraus besser zu erkennen. So hätten marginalisierte Gruppen das Interesse, ihre Position im gesellschaftlichen Gesamtsystem zu finden (Eagleton 2000: 114f.). „Genau in diesem Sinn erweist sich das Schreckgespenst des Relativismus als irrelevant: Denn die Behauptung, daß jede Erkenntnis von einem spezifischen gesellschaftlichen Standpunkt herrührt, impliziert nicht, daß ein gesellschaftlicher Standpunkt für diese Zwecke so gut ist wie ein anderer" (Eagleton 2000: 115). Dieses Argument, dass es herausgehobene Erkenntnispositionen gebe, wurde auch später von anderen Theoretiker*innen wie zum Beispiel der feministischen Standpunkt-Theorie - hier aber für die Frauen - aufgenommen (Haraway 1995 [1988], Harding 1991 [1986]).

Damit können zwei Umgangsweisen mit dem Zirkularitätsproblem benannt werden, die helfen, den eigenen Wissensanspruch zu begründen. Ein erstes Argument lautet, dass kontextualisiertes Wissen nicht gleichzeitig falsch sein müsse. So lautete die herkömmliche Begründung für den herausgehobenen Wissensanspruch von wissenschaftlichem Wissen, dass dieses objektiv sei und nicht erklärt zu werden brauche, da es logischkausal begründet sei. Der soziale Kontext seiner Produktion habe keinen Einfluss auf seinen Inhalt. Damit würde sich jeder Ansatz, der soziale Kontextfaktoren für die Erklärung wissenschaftlichen Wissens einführe, selbst widerlegen, da dann auch die eigenen Wissensansprüche durch den sozialen Kontext verzerrt und mithin falsch sein müssten. Nach Bloor (1991, 17f.) funktioniere dieses Argument aber nur, wenn davon ausgegangen werde, dass Kausalität mit Fehler gleichzusetzen sei. Dies sei aber nicht der Fall.

Auch die beiden Werturteilsstreite in der deutschen Soziologie machten deutlich, dass die wertbezogene Grundlage sozialwissenschaftlichen Wissens nicht die Wissenschaftlichkeit der Analyse beeinträchtige. Vielmehr sei die Wertbasiertheit gerade die Voraussetzung der sozialwissenschaftlichen Theoriebildung (Weber (1988 [1904]), Adorno (1993 [1969]). 
Die Wissenschaftskritik kritisierte gleichermaßen an dem herkömmlichen, naturwissenschaftlich geprägten Wissenschaftsmodell den entkörperten "Blick vom Nirgendwo“ (Haraway 1995 [1988]), also die Annahme, dass eine Beobachtung der sozialen Wirklichkeit möglich sei, ohne dass die eigene Erkenntnisposition einen Einfluss darauf habe. Im Umkreis dieser wertbasierten Wissenschaftsauffassung ist nach Eagleton (2000) auch die Ideologiekritik zu verorten, da es eines ihrer Anliegen sei, darauf zu verweisen, dass Ideen und damit Wissensformen in praktischen Verhältnissen gründeten (vgl. Eagleton 2000, 85f.).

Eine zweite Umgangsweise mit dem Problem der Zirkularität besteht in der Annahme, dass es in der Gesellschaft Positionen gebe, die eher dafür prädestiniert seien, die gesellschaftliche Wirklichkeit weniger verzerrt wahrzunehmen. Eine solch herausgehobene Erkenntnisposition schlägt auch die Wissenssoziologie vor. Im Gegensatz zu Lukács und der feministischen Standpunkt-Theorie werden hierfür jedoch die Intellektuellen und nicht die Arbeiterklasse oder die Frauen prädestiniert. In seinem Buch „Ideologie und Utopie“ stellt Karl Mannheim (1985 [1929]) die These von der Seinsverbundenheit des Denkens auf und behauptet die Abhängigkeit des Denkens von seinem sozialen Standort. Damit setzt er jegliches Wissen einem totalen Ideologieverdacht aus. Das heißt, dass, entgegen zum Beispiel bei Karl Marx, der die Ideologieträchtigkeit nur auf die bürgerliche Klasse beschränkte, grundsätzlich allen sozialen Schichten ein privilegierter Zugang zur „Wahrheit" abgesprochen wird. Die Aufgabe der Wissenssoziologie bestehe nun darin, die in den unterschiedlichen Perspektiven dennoch enthaltenen Wahrheitsmomente zu einer Synthese zusammenzuführen. Für diese Aufgabe prädestiniert sei die „freischwebende Intelligenz", da diese in der Moderne durch ihre minimalen Bindungen an eine soziale Schicht am wenigsten ideologieanfällig sei (Mannheim 1985 [1929], 11f.). Mannheim verband mit dieser Konzeption auch politische Ziele, nämlich über die Wissenssoziologie ideologisches Denken zu überwinden und den zerstrittenen politischen Parteien der Weimarer Republik zu einem gemeinsamen Verständnis ihrer Situation zu verhelfen (Kettler und Meja 1999). Die Kritik an Mannheims Konzept erfolgte von Seiten der Kritischen Theorie dahingehend, dass die Unterstellung eines "totalen Ideologieverdachts“ nur die Relativität und damit Unverbindlichkeit jedes Wissens darstelle und die sozialen Ungleichheiten nicht zu analysieren vermöge (vgl. Wiggershaus 1997, 65). Auch Eagleton 
kritisiert an Mannheims Ideologiebegriff, dass dieser zu flach sei. Ein gehaltvoller Ideologiebegriff verbinde diesen mit Machtkämpfen (Eagleton 2000, 130f.). Ähnlich fordert Rehmann (2008, 33), dass die moderne Ideologietheorie die Machtkämpfe einer herrschenden Klasse untersuchen müsse.

Ideologiekritik kann sich in der Tradition der Logik der Sozialwissenschaften (Ritsert 1996) verorten und auf einen Letztbegründungsanspruch ihrer Aussagen verzichten. Das Entscheidende an der Ideologiekritik ist damit nicht ihre besondere Erkenntnisposition, sondern ihre spezifische Aufgabe, Wissensformen und Legitimierungsstrategien von Macht und Herrschaft empirisch zu untersuchen.

\section{Theoretische Begriffe und Bedeutungsfixierung}

Ein weiteres Problem der Wissenschaftstheorie, das Bezüge zur Ideologietheorie aufweist und im Folgenden mit dem Begriff der "Verdinglichung" in Verbindung gebracht wird, ist das Verhältnis von Empirie und Theorie. Bei diesem wissenschaftstheoretischen Problem, das auf einer hierarchischen Wissenschaftsauffassung beruht, stellt sich die Frage, wie empirische Beobachtungen über die Formulierung von Beobachtungssätzen zur Ableitung von theoretischen Aussagen führen oder wie aus einer Theorie abgeleitete theoretische Aussagen empirisch geprüft werden können. Die Möglichkeit, theoretische Aussagen in der Empirie zu begründen, wurde vor allem durch die Theoreme der post-positivistischen Wende in Frage gestellt (Ritsert 1996, 290ff., Felt et al. 1995, 101ff.). Dabei spielen drei Thesen eine Rolle, die im Folgenden mit Rückgriff auf die Ausführungen von Franz von Kutschera (1982) zur Erkenntnis- und Wissenschaftstheorie skizziert werden sollen: (1) die These von der Theoriegeladenheit der Beobachtung, (2) die These von der Unterbestimmtheit von Theorien und (3) die Duhem-Quine-These.

(1) Die These von der Theoriegeladenheit der Beobachtung problematisiert das Verhältnis von Erfahrung und Beobachtungssätzen. Demnach gibt es keine unvoreingenommene Beobachtung. In die Formulierung von Beobachtungssätzen und die Bewertung eines Faktes (= ob ein empirischer Sachverhalt zutrifft) fließen stets apriorische Komponenten ein, also allgemeine Annahmen und Glaubenssätze über die Beschaffenheit der Welt (Kutschera 1982, 455ff.). 
(2) Die These von der Unterbestimmtheit von Theorien problematisiert das Verhältnis von Beobachtungssätzen bzw. empirischen Sätzen zu theoretischen Sätzen. Demnach lassen sich theoretische Aussagen nicht vollständig von Beobachtungsaussagen herleiten. Es können in vielen Fällen mehrere Interpretationen über die Zusammenhänge von Fakten gegeben werden und es lässt sich nicht entscheiden, welche Interpretation die richtige ist. Zudem gibt es mehrere Kriterien, sich für eine Theorie (als die "wahre") zu entscheiden. Diese Kriterien, wie zum Beispiel Informativität, Einheitlichkeit und Einfachheit, können auch miteinander in Konkurrenz treten (Kutschera 1982, 461ff.).

(3) Die Duhem-Quine-These steht in engem Zusammenhang mit dem Falsifikationsproblem. Falsifikation heißt, eine theoretische Annahme als falsch zu verwerfen. Die Frage ist, wann Beobachtungen, die einer theoretischen Annahme widersprechen, als Anlass dafür genommen werden, diese theoretische Annahme abzulehnen. So könnten auch Randbedingungen oder Kontextfaktoren dafür verantwortlich gemacht werden, dass die empirische Beobachtung der theoretischen Annahme widerspricht. Nach der Duhem-Quine-These lässt sich nun noch nicht einmal entscheiden, was getan werden muss, wenn es widersprechende Beobachtungen gibt: Die These besagt, dass im Prinzip nicht einzelne Aussagen, sondern, wenn auch implizit, immer ganze Theorien getestet werden, da die einzelnen Aussagen im Gesamtzusammenhang der Theorie stehen. Somit kann bei widersprechenden Beobachtungen nicht entschieden werden, ob nur die abgeleitete theoretische Aussage oder die gesamte Theorie falsch ist. Im Endeffekt kann es darum keine Beobachtung bzw. Erfahrung geben, an der Theorien endgültig scheitern (Kutschera 1982, 512ff.).

Diese drei Thesen der post-positivistischen Wende in der Wissenschaftstheorie begründeten aus einer theoretischen Perspektive das - bereits im vorangegangenen Abschnitt in Bezug auf die sozialen Kontextfaktoren und die Zirkularität wissenschaftlicher Erkenntnis diskutierte - Relativismus-Problem. Demnach kann es mehrere miteinander konkurrierende, aber inkommensurable Theorien geben und kein Kriterium, welche Theorie gilt und welche nicht.

Statt einer logischen und empirischen Begründung wurden soziale Faktoren für die Auswahl von wissenschaftlichen Theorien verantwortlich gemacht. Wegweisend waren hier die wissenschaftshistorischen Studien von Thomas S. Kuhn. In seinem Buch „Die Struktur wissenschaftli- 
cher Revolutionen“ (Kuhn (1999 [1962]) belegt er anhand von wissenschaftshistorischen Beispielen aus der Physik, dass in den Naturwissenschaften der wissenschaftliche Forschungsprozess nicht durch eine Akkumulation wahrer Aussagen, die sich allein durch ihre empirische Basis begründen, vorangetrieben werde. Kuhn betont den Einfluss von sozialen Überzeugungen innerhalb der Wissenschaftlergemeinde (Paradigmata) über die Form legitimer wissenschaftlicher Praxis, die zentralen Problemstellungen sowie Sichtweisen auf die Natur, ob eine Theorie akzeptiert oder abgelehnt wird.

Das wissenschaftstheoretische Problem des Verhältnisses von empirischen zu theoretischen Aussagen kann mit dem Konzept der „Verdinglichung" aus der Ideologiekritik in Verbindung gebracht werden. Verdinglichung lässt sich als ein Vorgang beschreiben, bei dem komplexe gesellschaftliche Verhältnisse, geblackboxt' werden. Dies ist dem Vorgehen ähnlich, empirische Begriffe unter einem allgemeinen Begriff zu fassen. ${ }^{4}$ Im Folgenden wird das Konzept der Verdinglichung in der Ideologiekritik kurz skizziert. Dabei wird deutlich, wie sich im Laufe der Entwicklung dieser Gedankenfigur der Schwerpunkt von der gesellschaftlichen Praxis auf die Begriffsbildung verschiebt.

Karl Marx beschrieb Verdinglichung auf der Ebene der gesellschaftlichen Praxis als „Warenfetischismus“. Im Produkt der Ware würden die gesellschaftlichen Verhältnisse, die zu ihrer Produktion führten, nicht deutlich und es schiene, als ob die Menschen über den Austausch von Waren auf dem Markt miteinander in Beziehung treten und nicht über deren Produktion in Arbeitsorganisationen. Dadurch könne der gesamtgesellschaftliche Zusammenhang nur noch bruchstückhaft wahrgenommen werden; er würde naturalisiert und schiene unveränderbar (Marx 1953 [1867]). Laut Rehmann (2008, 67ff.) erweiterte Lukács diese Gedankenfigur bereits hin zu einer "Universalkategorie“, so dass Verdinglichung sich auf jede Tätigkeit des Menschen bezog, die ihm als etwas Fremdes und Unabhängiges gegenübergestellt wird. Dieses problematische Verhältnis des Menschen zu seinen Produkten könne dadurch gelöst werden, das Ganze der gesellschaftlichen Totalität zu erkennen, um sich darin wiederzufinden (vgl. Rehmann 2008; Eagleton 2000, 117ff.).

4 Zur Gedankenfigur der „Black Box“ von wissenschaftlichen Ergebnissen vgl. Latour 1987. 
Die Figur der Verdinglichung findet sich dann bei Adorno in seiner Kritik des identifizierenden Denkens wieder (vgl. Eagleton 2000, 148ff; Ritsert 2002, 88f.). Adorno zieht eine Parallele zwischen dem Warenaustausch und dem ideologischen Denken. So wie im Warenaustausch Dinge gleichgesetzt werden, die eigentlich inkommensurabel seien, reduziere das identifizierende (= ideologische) Denken Dinge auf ihren Begriff. Durch die Leugnung dessen, was nicht vom Begriff erfasst werde ("das Nichtidentische“, vgl. Adorno 1975 [1966]), würden die Dinge universell austauschbar.

Das Problem des identifizierenden Denkens bzw. der Bedeutungsfixierung ist mit dem Problem des unbestimmten Verhältnisses von theoretischen und empirischen Sätzen vergleichbar. Die Kritik an der Bedeutungsfixierung bezieht sich darauf, ein Determinationsverhältnis zwischen Beobachtung und (allgemeinem) Begriff anzunehmen. Gerade dieses Determinationsverhältnis wurde durch die Thesen der post-positivistischen Wende in Frage gestellt. Insbesondere Adorno hat in seiner Negativen Dialektik als Gegenmittel zur Bedeutungsfixierung das beständige Ringen um Wahrheit, ein Offenhalten gegenüber Bedeutungen genannt (Adorno (1975 [1966]).

Auch in der Wissenschaftstheorie gibt es Positionen, welche Theorienpluralismus und Offenheit auch gegenüber unkonventionellen Methoden als positive Werte ansehen. Hier ist vor allem Paul Feyerabend (1976) und sein Buch „Wider dem Methodenzwang - Skizze einer anarchistischen Erkenntnistheorie" bekannt geworden, in dem er ebenso davon ausgeht, dass jede Theorie ihre Grenzen habe und es keine Theorie gebe, die alle Tatsachen erklären könne (Feyerabend 1976, 33). Der Anschein, eine Theorie würde mit den Tatsachen übereinstimmen, entstehe nur, da alle Alternativen, die widersprechende Tatsachen produzieren könnten, ausgeschaltet und nur die Tatsachen ausgearbeitet würden, welche die Theorie stützen. Eine Theorie werde dann zu einem Mythos und zu Ideologie, die für jeden Fall eine Erklärung bereitstelle (Feyerabend 1976, 49ff.). Objektive Erkenntnis benötige jedoch unterschiedliche Ideen. Feyerabend drängt auf Offenheit in der Erkenntnis und auf Kreativität des Forschenden. Neben die Anwendung von Methoden setzt Feyerabend $(1976,56)$ einen beständigen Vergleich und Kontrast von Ideen.

Doch zurück zum Begriff der Verdinglichung bei Adorno. Adornos Auffassung von verdinglichendem Denken und von Ideologie kritisiert Eagleton folgendermaßen: „Dieser Auffassung nach wäre das Gegenteil 
von Ideologie nicht Wahrheit oder Theorie, sondern Differenz oder Heterogenität“ (Eagleton 2000, 148). Und weiter: „Adorno und die Seinen liefern uns die Ideologie als Pappkameraden im Stil poststrukturalistischer Theoretiker, für die sich Ideologie ausnahmslos um metaphysische Absoluta und transzendentale Grundlagen dreht" (Eagleton 2000, 150). Damit deutet Eagleton an, dass die Strukturierung der Erfahrung durch die Praxis (wie zum Beispiel den Warenaustausch) und die Begriffsbildung zur Erfassung der gesellschaftlichen und nichtgesellschaftlichen Wirklichkeit nicht in eins gesetzt werden dürfen. Die ursprüngliche Bedeutung des "Warenfetischismus", nämlich dass in der Alltagserfahrung, die nur Ausschnitte erfassen kann, bestimmte soziale Verhältnisse verdinglicht erscheinen, wird in der Kritik an der Bedeutungsfixierung auf eine rein intellektuelle Ebene verschoben.

Damit kann abschließend bemerkt werden: Bedeutungsfixierungen sind zumindest als vorläufige Verallgemeinerungen das Ergebnis wissenschaftlicher Arbeit. Sie werden dann zu einem Problem, wenn sie nicht mehr hinterfragt und alternative Sichtweisen nicht (kritisch) wertgeschätzt werden. Dieses Ringen um Wahrheit scheint es bei Ideologien nicht zu geben.

Bei ideologischem Denken kommt jedoch noch ein weiterer Aspekt hinzu, wie die Paraphrase der Position von Althusser bei Eagleton (2000, 160ff.) verdeutlicht: Jedes Denken entlang von Begriffen enthalte unbewusst eine Problematik, die es untermauere. Nicht jede Problematik sei an sich ideologisch: „Eine ideologische Problematik dreht sich um gewisse vielsagende Auslassungen und Leerstellen und ist so konstruiert, daß die Fragen, die gestellt werden können, schon bestimmte Antworten vorgeben" (Eagleton 2000, 161; Herv. B. P.). Wissenschaftliche Problematiken seien hingegen durch Offenheit gekennzeichnet (ebd.). Ideologien legen damit mögliche Problemlösungen nahe. Darüber hinaus definieren sie die Akteure, die an der Problemlösung beteiligt sind. Sie verteilen und legitimieren Macht. Nach Eagleton $(2000,69)$ legitimiert sich eine herrschende Klasse jedoch nie allein nur durch ideologische Mittel, sondern vielmehr durch materielle Mittel. Damit sind Ideologien nicht mit Macht gleichzusetzen und sind nicht das alleinige Machtmittel. Es gibt weitere, nichtsprachliche Machtmittel. Diese alle unter den Ideologiebegriff fassen zu wollen, würde den Begriff überdehnen und sein analytisches Potenzial schwächen. 
Die Problemdimension, wie von Wissensarbeiter*innen produzierte Wissensformen in die Alltagserfahrung eindringen, könnte dann empirisch geprüft werden. Die Frage wäre dann, welche Gründe dafür angegeben werden können, dass Handlungs- und Verhaltensweisen der Menschen ihren (unterstellten oder von ihnen selbst artikulierten) Interessen zuwiderlaufen. So könnten neben ideologischen Wissensformen auch materielle Verhältnisse für ein widersprüchliches Verhalten verantwortlich sein, das den Akteuren durchaus bewusst ist.

\section{$4 \quad$ Schlussfolgerung}

In dem Beitrag wurden Bezüge von Ideologiekritik und Wissenschaftstheorie sowie zur Soziologie wissenschaftlichen Wissens aufgezeigt. Dabei wurde gezeigt, dass die Aporien im Ideologiebegriff durch die Wissenschaftstheorie der Sozialwissenschaften aufgeklärt werden können. Dies betrifft zum einen die Epistemologie als auch die gesellschaftlichen Bedingungen, die zur Entstehung von Ideologien beitragen.

Aus epistemologischer Sicht stellt die Ideologiekritik einen empirischen sozialwissenschaftlichen Ansatz dar. Die Probleme und Aporien, die in der Diskussion des Ideologiebegriffs auftauchen, sind weniger im Begriff selbst begründet, sondern in den Problemen, die sich jeder sozialwissenschaftlichen Forschung stellen. Wenn die Ideologiekritik sich als eine sozialwissenschaftliche Forschungspraxis begreift, braucht sie nicht in die Diskussion des Ideologiebegriffs Probleme aufzunehmen, die eigentlich der Forschungspraxis zuzuordnen sind. Weiterhin wurde dafür plädiert, den Ideologiebegriff auf eine bestimmte, von anderen abgrenzbare Wissensform zu fokussieren. Ansatzpunkt hierfür liefern die Überlegungen in der Wissenschaftstheorie der Sozialwissenschaften und der Wissenschaftskritik, Ideologiebildungen im Wissenschaftssystem zu vermeiden und damit Wissensformen zu ermöglichen, die weniger ideologisch sind.

Hinsichtlich der Unterscheidung von wissenschaftlichem und ideologischem Wissen lassen sich einige abschließende Bemerkungen machen. Eine Problematisierung ist konstitutiv für wissenschaftliches und ideologisches Wissen. Ein Unterschied besteht in den präferierten Lösungsmustern. Sozialwissenschaftliche Forschung ist eher vorsichtig bei 
der Ableitung von Handlungsempfehlungen. Ideologisches Wissen präferiert ein Lösungsmuster und legitimiert seine Auswahl. Pauschalisierungen finden notgedrungen auch bei wissenschaftlichen Wissen statt, denn Wissenschaft will verallgemeinerbares Wissen herstellen. Bei Ideologien ist das Ringen um die Wahrheit weniger gegeben. Es wird eher mit allgemeinen Bildern und Narrationen gearbeitet.

Sowohl Wissenschaft als auch Ideologiekritik haben einen Ort in der Gesellschaft. Sie unterliegen als wissenschaftliche Praktiken bestimmten normativen Anforderungen, da sie „wahres“ Wissen über Wissen hervorbringen wollen. Die mangelnde Positionierung des Wissenssubjekts ist durch die Wissenschaftskritik insbesondere an den Naturwissenschaften als „Blick von Nirgendwo“ kritisiert worden, der bestimmte Wissensansprüche weniger angreifbar macht und selbst als ideologisch bezeichnet werden könnte.

Sowohl für wissenschaftliches als auch für ideologisches Wissen können gesellschaftliche Bedingungen angegeben werden. Neben den tatsächlichen Produzenten, den Intellektuellen, sind dabei die institutionellen Infrastrukturen wie Universitäten, Think Tanks und Stiftungen zu nennen (vgl. Plehwe, Walpen und Neunhöffer 2006).

Eagleton (2000, 59ff.) bezeichnet Ideologien als handlungsorientierte Überzeugungskomplexe. Damit können Ideologien als eine Wissensform angesehen werden, die in der Nähe von Macht und Herrschaft verortet ist. Ideologien präferieren ein bestimmtes Lösungsmuster einer bestimmten Akteursgruppe. Ideologien sind damit eher dem begrifflichen Denken als dem unbewussten "tacit knowledge“ zuzuordnen. Sie legitimieren Machtpositionen in der Gesellschaft, was auf der individuellen Ebene auch bedeuten kann, bestimmte Verhaltenserwartungen zu erfüllen. Dieses von Rehmann (2008) als „freiwillige Unterwerfung“ bezeichnete Problem der durch Ideologien beeinflussten Subjekt- und Identitätskonstruktionen und die Rolle, welche der Alltagserfahrung dabei zukommt, konnte im Rahmen dieses Aufsatzes nicht nachgegangen werden.

Um auf die eingangs gestellte Frage zurückzukommen, inwiefern der Ideologiebegriff dazu geeignet sein kann, über die Macht der jeweiligen Diskursposition aufzuklären: Bei der Analyse von Technik- und Risikokonflikten müssten dann nicht nur allein Diskurse analysiert werden. Vielmehr müssten die Diskurspositionen als ideologische Wissensformen und somit als ein Machtmittel in Zusammenhang mit anderen, nicht wissensförmigen Machtmitteln analysiert werden, die Macht und Herrschaft 
begründen. So könnten Macht und Durchsetzungskraft von Diskurspositionen bestimmt werden.

Der Praxisbezug eines solchen Ansatzes wäre, von einer Trennung von Wissenschaft und Politik auszugehen und nicht in politische Machtkämpfe einzugreifen. Dennoch sollte die wertbezogene Positionierung der Wissenschaftler*in offengelegt werden, gegen welche bestehenden Macht- und Herrschaftsstrukturen sich die Analyse kritisch richtet.

\section{Literatur}

Adorno, Theodor W. 1975 [1966]. Negative Dialektik. Frankfurt am Main: Suhrkamp.

Adorno, Theodor W. 1993 [1969]. „Zur Logik der Sozialwissenschaften“. In Der Positivismusstreit in der deutschen Soziologie, Theodor W. Adorno et al., 125-143. Frankfurt am Main: Luchterhand.

Bloor, David. 1991. Knowledge and Social Imagery. Chicago, London: University of Chicago Press.

Böhme, Günther, und Klaus Potyka. 1995. Erfahrung und Wissenschaft und Alltag. Eine analytische Studie über Begriffe, Gehalt und Bedeutung eines lebensbegleitenden Phänomens. Idstein: Schulz-Kirchner.

Eagleton, Terry. 2000. Ideologie. Eine Einführung. Stuttgart, Weimar: Metzler.

Dilthey, Wilhelm. 1974 [1900]. „Die Entstehung der Hermeneutik“. In Die Geistige Welt. Einleitung in die Philosophie des Lebens. Wilhelm Dilthey, Gesammelte Schriften Bd. V. Stuttgart, Göttingen: Vandenhoeck \& Ruprecht.

Felt, Ulrike, Helga Nowotny und Klaus Taschwer. 1995. Wissenschaftsforschung. Eine Einführung. Frankfurt am Main, New York: Campus.

Feyerabend, Paul. 1976. Wider dem Methodenzwang. Skizze einer anarchistischen Erkenntnistheorie. Frankfurt am Main: Suhrkamp

Haraway, Donna. 1995 [1988]. „Situiertes Wissen. Die Wissenschaftsfrage im Feminismus und das Privileg einer partialen Perspektive“. In Die Neuerfindung der Natur. Primaten, Cyborgs und Frauen. Donna Haraway herausgegeben von Carmen Hammer und Immanuel Stieß, 73-97. Frankfurt am Main, New York: Campus.

Harding, Sandra. 1991 [1986]. Feministische Wissenschaftstheorie. Zum Verhältnis von Wissenschaft und sozialem Geschlecht. Hamburg: Argument.

Herkommer, Sebastian. 2004. Metamorphosen der Ideologie. Zur Analyse des Neoliberalismus durch Pierre Bourdieu und aus marxistischer Perspektive. Hamburg: VSA.

Kettler, David und Volker Meja. 1999. „Karl Mannheim“. In Klassiker der Soziologie. Bd.1. Von Auguste Comte bis Norbert Elias, herausgegeben von Dirk Kaesler, 296-314. München: C.H. Beck.

Knoblauch, Hubert. 2010. Wissenssoziologie. Konstanz: UVK. 
Kuhn, Thomas S. 1999 [1962]. Die Struktur wissenschaftlicher Revolutionen. Frankfurt am Main: Suhrkamp.

Kutschera, Franz von. 1982. Grundfragen der Erkenntnistheorie. Berlin, New York: de Gruyter.

Latour, Bruno. 1987. Science in action. How to follow scientists and engineers through society. Cambridge: Cambridge.

Marx, Karl und Friedrich Engels. 1953 [1857]. „Die deutsche Ideologie. Kritik der neuesten deutschen Philosophie in ihren Repräsentanten Feuerbach, B. Bauer und Stirner, und des deutschen Sozialismus in seinen verschiedenen Propheten". In Bücherei des Marxismus-Leninismus, Bd. 29. Berlin/DDR: Dietz.

Marx, Karl. 1953 [1867]. Das Kapital. Kritik der politischen Ökonomie. Band I. Berlin/DDR: Dietz.

Mannheim, Karl. 1985 [1929]. Ideologie und Utopie. Frankfurt am Main: Klostermann.

Peuker, Birgit. 2010. Der Streit um die Agrar-Gentechnik. Perspektiven der Akteur-Netzwerk-Theorie. Bielefeld: transcript.

Plehwe, Dieter, Bernhard Walpen, und Gisela Neunhöffer. 2006. „Introduction: Reconsidering Neoliberal Hegemony“. In Neoliberal Hegemony. A Global Critique, herausgeben von Dieter Plehwe, Bernhard Walpen und Gisela Neunhöffer, 1-24. London, New York: Routledge.

Rehmann, Jan. 2008. Einführung in die Ideologietheorie. Hamburg: Argument.

Ritsert, Jürgen. 2002. Ideologie. Theoreme und Probleme der Wissenssoziologie. Münster: Westfälisches Dampfboot.

Ritsert, Jürgen. 1996. Einführung in die Logik der Sozialwissenschaften. Münster: Westfälisches Dampfboot.

Weber, Max. 1988 [1904]. „Die „Objektivität” sozialwissenschaftlicher und sozialpolitischer Erkenntnis." In Max Weber, Gesammelte Aufsätze zur Wissenschaftslehre, herausgegeben von Johannes Winckelmann, 146-214. Tübingen: J. C. B. Mohr.

Wiggershaus, Rolf. 1997. Die Frankfurter Schule. Geschichte, Theoretische Entwicklung, Politische Bedeutung. München, Wien: dtv.

\section{Open Access}

Dieser Beitrag erscheint unter der Creative-Commons-Lizenz CC-BY-SA 4.0:

https://creativecommons.org/licenses/by-sa/4.0/ 